\title{
More results on a functional generalization of the Cauchy-Schwarz inequality
}

\author{
Mohammad Masjed-Jamei ${ }^{1}$ and Nawab Hussain ${ }^{2 *}$
}

${ }^{*}$ Correspondence:

nhusain@kau.edu.sa

2Department of Mathematics, King

Abdulaziz University, P.O. Box 80203,

Jeddah, 21589, Saudi Arabia

Full list of author information is

available at the end of the article

\begin{abstract}
By using a specific functional property, some more results on a functional generalization of the Cauchy-Schwarz inequality, such as an extension of the pre-Grüss inequality and a refinement of the Cauchy-Schwarz inequality via the generalized Wagner inequality, are given for both discrete and continuous cases. MSC: 26D15; 26D20

Keywords: functional generalization of the Cauchy-Bunyakovsky-Schwarz inequality; Chebyshev functional; extension of the pre-Grüss inequality; generalized Wagner inequality; refinement of the Cauchy-Schwarz inequality
\end{abstract}

\section{Introduction}

It is well known that the discrete version of the Cauchy-Schwarz inequality [1]

$$
\left(\sum_{i=1}^{n} a_{i} b_{i}\right)^{2} \leq \sum_{i=1}^{n} a_{i}^{2} \sum_{i=1}^{n} b_{i}^{2} \quad\left(a_{i}, b_{i} \in \mathbf{R}\right),
$$

and its integral representation in the space of continuous real-valued functions $C([a, b], \mathbf{R})$, i.e., the Cauchy-Bunyakovsky inequality [1]

$$
\left(\int_{a}^{b} f(x) g(x) d x\right)^{2} \leq \int_{a}^{b} f^{2}(x) d x \int_{a}^{b} g^{2}(x) d x
$$

play an important role in different branches of modern mathematics such as Hilbert space theory, classical real and complex analysis, numerical analysis, probability and statistics, qualitative theory of differential equations and their applications. To date, a large number of generalizations and refinements of the inequalities (1) and (2) have been investigated in the literature, e.g., [2-7].

Recently in [8], we have presented a functional generalization of the CauchyBunyakovsky-Schwarz inequality for both discrete and continuous cases as follows.

Theorem 1 Let $\left\{a_{i 1}, a_{i 2}, \ldots, a_{i m}\right\}_{i=1}^{n}$ and $\left\{b_{i 1}, b_{i 2}, \ldots, b_{i k}\right\}_{i=1}^{n}$ be real numbers for any $m, k \in \mathbf{N}$. If $F_{m}\left(x_{1}, x_{2}, \ldots, x_{m}\right)$ and $G_{k}\left(x_{1}, x_{2}, \ldots, x_{k}\right)$ are two arbitrary functions of $m$ and $k$ variables, then the following inequality holds:

$$
\left(\sum_{i=1}^{n} F_{m}\left(a_{i 1}, a_{i 2}, \ldots, a_{i m}\right) G_{k}\left(b_{i 1}, b_{i 2}, \ldots, b_{i k}\right)\right)^{2}
$$

(0) 2012 Masjed-Jamei and Hussain; licensee Springer. This is an Open Access article distributed under the terms of the Creative Commons Attribution License (http://creativecommons.org/licenses/by/2.0), which permits unrestricted use, distribution, and reproduction in any medium, provided the original work is properly cited. 


$$
\leq \sum_{i=1}^{n} F_{m}^{2}\left(a_{i 1}, a_{i 2}, \ldots, a_{i m}\right) \sum_{i=1}^{n} G_{k}^{2}\left(b_{i 1}, b_{i 2}, \ldots, b_{i k}\right) .
$$

Moreover, for the integral form of the above inequality, if $\left\{f_{j}(x)\right\}_{j=1}^{m}$ and $\left\{g_{j}(x)\right\}_{j=1}^{k}$ are real functions on $[\alpha, \beta]$, then

$$
\begin{aligned}
& \left(\int_{\alpha}^{\beta} F_{m}\left(f_{1}, f_{2}, \ldots, f_{m}\right) G_{k}\left(g_{1}, g_{2}, \ldots, g_{k}\right) d x\right)^{2} \\
& \quad \leq \int_{\alpha}^{\beta} F_{m}^{2}\left(f_{1}, f_{2}, \ldots, f_{m}\right) d x \int_{\alpha}^{\beta} G_{k}^{2}\left(g_{1}, g_{2}, \ldots, g_{k}\right) d x .
\end{aligned}
$$

Thus, inequalities (3) and (4) are respectively generalizations of the discrete and continuous Cauchy-Bunyakovsky-Schwarz inequalities for $m=k=1, F_{1}\left(a_{i 1}\right)=a_{i 1}$ and $G_{1}\left(b_{i 1}\right)=b_{i 1}$ in (3) and $F_{1}\left(f_{1}\right)=f_{1}(x)$ and $G_{1}\left(g_{1}\right)=g_{1}(x)$ in $(4)$.

Also, the equality holds if in (3) $F_{m}\left(a_{i 1}, a_{i 2}, \ldots, a_{i m}\right)=r G_{k}\left(b_{i 1}, b_{i 2}, \ldots, b_{i k}\right)$ where $r$ is constant and $F_{m}\left(f_{1}, f_{2}, \ldots, f_{m}\right)=R G_{k}\left(g_{1}, g_{2}, \ldots, g_{k}\right)$ in (4) where $R$ is constant.

The aim of this paper is to extend the results of the above-mentioned theorem by using a specific functional property.

\section{On the generalized Cauchy-Schwarz inequality}

Suppose that $\mathbf{L}$ is a linear functional applied to the arbitrary function $h(x)$, and then consider the following special function:

$$
H(x ; h, \lambda)=h(x)+\lambda \mathbf{L}(h) \quad(\lambda \in \mathbf{R} \backslash\{0\}) .
$$

One of the important properties of $H(x ; h, \lambda)$ is that

$$
\begin{aligned}
\mathbf{L}(H) & =\mathbf{L}(h+\lambda \mathbf{L}(h))=\mathbf{L}(h)+\lambda \mathbf{L}(h) \mathbf{L}(1) \\
& =(1+\lambda \mathbf{L}(1)) \mathbf{L}(h)=K \mathbf{L}(h) \quad(K:=1+\lambda \mathbf{L}(1)) .
\end{aligned}
$$

This means that only for once one can take an arbitrary linear functional on both sides of the function (5) and not more because

$$
\mathbf{L}(\mathbf{L}(H))=\mathbf{L}(K \mathbf{L}(h))=K \mathbf{L}(h) \mathbf{L}(1)=K^{*} \mathbf{L}(h) \quad\left(K^{*}:=K \mathbf{L}(h) ; K:=1+\lambda \mathbf{L}(1)\right) .
$$

More generally, the property (6) can be considered for two special functions:

$$
\begin{cases}H_{1}\left(x ; h_{1}, \lambda_{1}\right)=h_{1}(x)+\lambda_{1} \mathbf{L}\left(h_{1}\right) & \left(\lambda_{1} \in \mathbf{R} \backslash\{0\}\right), \\ H_{2}\left(x ; h_{2}, \lambda_{2}\right)=h_{2}(x)+\lambda_{2} \mathbf{L}\left(h_{2}\right) & \left(\lambda_{2} \in \mathbf{R} \backslash\{0\}\right),\end{cases}
$$

as

$$
\begin{aligned}
\mathbf{L}\left(H_{1} H_{2}\right) & =\mathbf{L}\left(\left(h_{1}+\lambda_{1} \mathbf{L}\left(h_{1}\right)\right)\left(h_{2}+\lambda_{2} \mathbf{L}\left(h_{2}\right)\right)\right) \\
& =\mathbf{L}\left(h_{1} h_{2}\right)+K^{* *} \mathbf{L}\left(h_{1}\right) \mathbf{L}\left(h_{2}\right) \quad\left(K^{* *}:=\lambda_{1}+\lambda_{2}+\mathbf{L}(1) \lambda_{1} \lambda_{2}\right) .
\end{aligned}
$$


By using the aforesaid functional property, many classical inequalities such as Chebyshev, Stefensen and Aczel inequalities have been generalized in [9]. Here we wish to apply the property (8) to extend the results of Theorem 1. For this purpose, we first express the following lemma.

Lemma 2 Let the linear functional $\mathbf{L}$ be defined in such a way that $\mathbf{L}\left(h^{2}(x)\right) \geq 0$ for any arbitrary function $h(x)$, and $H_{1}, H_{2}$ be defined as (7). Then the following inequality holds:

$$
\mathbf{L}^{2}\left(H_{1} H_{2}\right) \leq \mathbf{L}\left(H_{1}^{2}\right) \mathbf{L}\left(H_{2}^{2}\right)
$$

Proof The proof is straightforward if one defines a positive quadratic polynomial $\mathbf{P}: \mathbf{R} \rightarrow$ $\mathbf{R}$ as

$$
\mathbf{P}\left(t ; H_{1}, H_{2}\right)=\mathbf{L}\left(\left(H_{1} t+H_{2}\right)^{2}\right)=\mathbf{L}\left(H_{1}^{2}\right) t^{2}+2 \mathbf{L}\left(H_{1} H_{2}\right) t+\mathbf{L}\left(H_{2}^{2}\right) \geq 0
$$

for any $t \in \mathbf{R}$, and then notes that the discriminant $\Delta$ of $\mathbf{P}$ must be negative.

It is now well known that the linear functional applied in the inequality (3) is $\mathbf{L}(\cdot)=\sum(\cdot)$ where $\mathbf{L}(1)=n$ and $\mathbf{L}\left(h^{2}\right) \geq 0$. So, by noting (7), if we define

$$
\left\{\begin{aligned}
H_{1, m}\left(a_{i 1}, a_{i 2}, \ldots, a_{i m}\right)= & h_{1, m}\left(a_{i 1}, a_{i 2}, \ldots, a_{i m}\right) \\
& +\lambda_{1} \sum_{i=1}^{n} h_{1, m}\left(a_{i 1}, a_{i 2}, \ldots, a_{i m}\right) \quad\left(\lambda_{1}=-p / n\right) \\
H_{2, k}\left(b_{i 1}, b_{i 2}, \ldots, b_{i k}\right)= & h_{2, k}\left(b_{i 1}, b_{i 2}, \ldots, b_{i k}\right) \\
& +\lambda_{2} \sum_{i=1}^{n} h_{2, k}\left(b_{i 1}, b_{i 2}, \ldots, b_{i k}\right) \quad\left(\lambda_{2}=-q / n\right)
\end{aligned}\right.
$$

where $p, q \in \mathbf{R}$, then we obtain

$$
\begin{aligned}
& \mathbf{L}\left(H_{1, m}\left(a_{i 1}, a_{i 2}, \ldots, a_{i m}\right)\right) \\
& \quad=\sum_{i=1}^{n}\left(h_{1, m}\left(a_{i 1}, a_{i 2}, \ldots, a_{i m}\right)-\frac{p}{n} \sum_{i=1}^{n} h_{1, m}\left(a_{i 1}, a_{i 2}, \ldots, a_{i m}\right)\right) \\
& \quad=(1-p) \sum_{i=1}^{n} h_{1, m}\left(a_{i 1}, a_{i 2}, \ldots, a_{i m}\right), \\
& \mathbf{L}\left(H_{1, m}^{2}\left(a_{i 1}, a_{i 2}, \ldots, a_{i m}\right)\right) \\
& \quad=\sum_{i=1}^{n} h_{1, m}^{2}\left(a_{i 1}, a_{i 2}, \ldots, a_{i m}\right)+\frac{p(p-2)}{n}\left(\sum_{i=1}^{n} h_{1, m}\left(a_{i 1}, a_{i 2}, \ldots, a_{i m}\right)\right)^{2} \geq 0,
\end{aligned}
$$

and

$$
\begin{aligned}
& \mathbf{L}\left(H_{1, m}\left(a_{i 1}, a_{i 2}, \ldots, a_{i m}\right) H_{2, k}\left(b_{i 1}, b_{i 2}, \ldots, b_{i k}\right)\right) \\
& =\sum_{i=1}^{n} h_{1, m}\left(a_{i 1}, a_{i 2}, \ldots, a_{i m}\right) h_{2, k}\left(b_{i 1}, b_{i 2}, \ldots, b_{i k}\right) \\
& \quad+\frac{p q-(p+q)}{n} \sum_{i=1}^{n} h_{1, m}\left(a_{i 1}, a_{i 2}, \ldots, a_{i m}\right) \sum_{i=1}^{n} h_{2, k}\left(b_{i 1}, b_{i 2}, \ldots, b_{i k}\right) .
\end{aligned}
$$


Hence, substituting these relations in the inequality (9) eventually yields

$$
\begin{aligned}
& \left(\sum_{i=1}^{n} h_{1, m}\left(a_{i 1}, \ldots, a_{i m}\right) h_{2, k}\left(b_{i 1}, \ldots, b_{i k}\right)\right. \\
& \left.\quad+\frac{p q-(p+q)}{n} \sum_{i=1}^{n} h_{1, m}\left(a_{i 1}, \ldots, a_{i m}\right) \sum_{i=1}^{n} h_{2, k}\left(b_{i 1}, \ldots, b_{i k}\right)\right)^{2} \\
& \leq\left(\sum_{i=1}^{n} h_{1, m}^{2}\left(a_{i 1}, \ldots, a_{i m}\right)+\frac{p(p-2)}{n}\left(\sum_{i=1}^{n} h_{1, m}\left(a_{i 1}, \ldots, a_{i m}\right)\right)^{2}\right) \\
& \quad \times\left(\sum_{i=1}^{n} h_{2, k}^{2}\left(b_{i 1}, \ldots, b_{i k}\right)+\frac{q(q-2)}{n}\left(\sum_{i=1}^{n} h_{2, k}\left(b_{i 1}, \ldots, b_{i k}\right)\right)^{2}\right)
\end{aligned}
$$

in which $\left\{a_{i 1}, a_{i 2}, \ldots, a_{i m}\right\}_{i=1}^{n}$ and $\left\{b_{i 1}, b_{i 2}, \ldots, b_{i k}\right\}_{i=1}^{n}$ are two sequences of real numbers, $m, k \in \mathbf{N}$ and $h_{1, m}\left(x_{1}, x_{2}, \ldots, x_{m}\right)$ and $h_{2, k}\left(y_{1}, y_{2}, \ldots, y_{k}\right)$ are two arbitrary functions of $m$ and $k$ variables. Moreover, the equality holds if in (10) $p=q$ and $h_{1, m}\left(a_{i 1}, a_{i 2}, \ldots, a_{i m}\right)=$ $r^{*} h_{2, k}\left(b_{i 1}, b_{i 2}, \ldots, b_{i k}\right)$ for the constant $r^{*}$.

Similarly, for the continuous case, the corresponding linear functional applied in (4) is $\mathbf{L}(\cdot)=\int_{\alpha}^{\beta}(\cdot) d x$, where $\mathbf{L}(1)=\beta-\alpha$ and again $\mathbf{L}\left(h^{2}\right) \geq 0$. So, if one sets

$$
\left\{\begin{array}{l}
H_{1, m}\left(f_{1}, f_{2}, \ldots, f_{m}\right)=h_{1, m}\left(f_{1}, f_{2}, \ldots, f_{m}\right)-\frac{p}{\beta-\alpha} \int_{\alpha}^{\beta} h_{1, m}\left(f_{1}, f_{2}, \ldots, f_{m}\right) d x \\
H_{2, k}\left(g_{1}, g_{2}, \ldots, g_{k}\right)=B_{k}\left(g_{1}, g_{2}, \ldots, g_{k}\right)-\frac{q}{\beta-\alpha} \int_{\alpha}^{\beta} h_{2, k}\left(g_{1}, g_{2}, \ldots, g_{k}\right) d x
\end{array}\right.
$$

in which $p, q \in \mathbf{R}$ and $\left\{f_{j}(x)\right\}_{j=1}^{m}$ and $\left\{g_{j}(x)\right\}_{j=1}^{k}$ are real functions on $[\alpha, \beta]$, then by using the inequality (9), one finally obtains

$$
\begin{aligned}
& \left(\int_{\alpha}^{\beta} h_{1, m}\left(f_{1}, \ldots, f_{m}\right) h_{2, k}\left(g_{1}, \ldots, g_{k}\right) d x\right. \\
& \left.\quad+\frac{p q-(p+q)}{\beta-\alpha} \int_{\alpha}^{\beta} h_{1, m}\left(f_{1}, \ldots, f_{m}\right) d x \int_{\alpha}^{\beta} h_{2, k}\left(g_{1}, \ldots, g_{k}\right) d x\right)^{2} \\
& \quad \leq\left(\int_{\alpha}^{\beta} h_{1, m}^{2}\left(f_{1}, \ldots, f_{m}\right) d x+\frac{p(p-2)}{\beta-\alpha}\left(\int_{\alpha}^{\beta} h_{1, m}\left(f_{1}, \ldots, f_{m}\right) d x\right)^{2}\right) \\
& \quad \times\left(\int_{\alpha}^{\beta} h_{2, k}^{2}\left(g_{1}, \ldots, g_{k}\right) d x+\frac{q(q-2)}{\beta-\alpha}\left(\int_{\alpha}^{\beta} h_{2, k}\left(g_{1}, \ldots, g_{k}\right) d x\right)^{2}\right) .
\end{aligned}
$$

The equality holds in (11) if $p=q$ and $h_{1, m}\left(f_{1}, f_{2}, \ldots, f_{m}\right)=R^{*} h_{2, k}\left(g_{1}, g_{2}, \ldots, g_{k}\right)$ for the constant $R^{*}$.

In this section we study two special cases of inequalities (10) and (11) which are remarkable.

Example 3 (An extension of the pre-Grüss inequality) Before deriving the main result, let us recall some initial comments. 
The space of $p$-power integrable functions on the interval $[a, b]$ is shown by $L^{p}[a, b]$ $(1 \leq p<\infty)$ with the norm

$$
\|f\|_{p}=\left(\int_{a}^{b}|f(t)|^{p} d t\right)^{1 / p}
$$

and the space of all essentially bounded functions on $[a, b]$ is denoted by $L^{\infty}[a, b]$ with the norm

$$
\|f\|_{\infty}=\operatorname{ess} \sup _{x \in[a, b]}|f(x)| .
$$

On the space $L^{2}[a, b]$, the inner product of Chebyshev type is defined by

$$
\langle f, g\rangle=\frac{1}{b-a} \int_{a}^{b} f(x) g(x) d x
$$

while the standard inner product is in the form

$$
(f, g)=(b-a)\langle f, g\rangle
$$

For two absolutely continuous functions $f, g:[a, b] \rightarrow \mathbf{R}$ such that $f, g, f g \in L^{1}[a, b]$, the Chebyshev functional is defined by

$$
\mathbf{T}\langle f, g\rangle=\langle f, g\rangle-\langle f, 1\rangle\langle 1, g\rangle=\frac{1}{b-a} \int_{a}^{b} f(x) g(x) d x-\frac{1}{(b-a)^{2}} \int_{a}^{b} f(x) d x \int_{a}^{b} g(x) d x .
$$

In 1882, Chebyshev [10] proved that if $f^{\prime}, g^{\prime} \in L^{\infty}[a, b]$, then

$$
|\mathbf{T}\langle f, g\rangle| \leq \frac{1}{12}(b-a)^{2}\left\|f^{\prime}\right\|_{\infty}\left\|g^{\prime}\right\|_{\infty}
$$

Later on, Grüss [11] in 1934 showed that

$$
|\mathbf{T}\langle f, g\rangle| \leq \frac{1}{4}\left(M_{1}-m_{1}\right)\left(M_{2}-m_{2}\right)
$$

where $m_{1}, m_{2}, M_{1}$ and $M_{2}$ are real numbers satisfying the conditions

$$
m_{1} \leq f(x) \leq M_{1} \quad \text { and } \quad m_{2} \leq g(x) \leq M_{2} \quad \text { for all } x \in[a, b]
$$

A remarkable point on the Chebyshev functional is that it can be represented in terms of the relation (8) as

$$
\langle f, g\rangle-\langle f, 1\rangle\langle 1, g\rangle=\langle f-\langle f, 1\rangle, g-\langle 1, g\rangle\rangle=\mathbf{L}\left(H_{1}(x ; f,-1) H_{2}(x ; g,-1)\right) \text {, }
$$

where

$$
H_{1}(x ; f,-1)=f(x)-\frac{1}{b-a} \int_{a}^{b} f(t) d t, \quad H_{2}(x ; g,-1)=g(x)-\frac{1}{b-a} \int_{a}^{b} g(t) d t,
$$


and

$$
\mathbf{L}(h)=\langle 1, h\rangle=\frac{1}{b-a} \int_{a}^{b} h(x) d x .
$$

Thus, substituting the above functions into the inequality (9) generates the well-known pre-Grüss inequality [1, p.296] as

$$
(\mathbf{T}\langle f, g\rangle)^{2} \leq \mathbf{T}\langle f, f\rangle \mathbf{T}\langle g, g\rangle \leq \frac{1}{16}\left(M_{1}-m_{1}\right)^{2}\left(M_{2}-m_{2}\right)^{2}
$$

On the other hand, the inequality (12) can be extended via the inequality (11). For this purpose, if the following generalized Chebyshev functional is defined as

$$
\mathbf{T}_{\lambda}\langle f, g\rangle=\langle f, g\rangle-\lambda\langle f, 1\rangle\langle 1, g\rangle \quad(\lambda \in \mathbf{R}),
$$

then, firstly, the aforesaid point is also valid for $\mathbf{T}_{\lambda}\langle f, g\rangle$ when $\lambda \leq 1$ so that we have

$$
\begin{aligned}
\langle f, g\rangle-\lambda\langle f, 1\rangle\langle 1, g\rangle= & \langle f-(1 \pm \sqrt{1-\lambda})\langle f, 1\rangle, g-(1 \pm \sqrt{1-\lambda})\langle 1, g\rangle\rangle \\
= & \frac{1}{b-a} \int_{a}^{b}\left(f(x)-\frac{(1 \pm \sqrt{1-\lambda})}{b-a} \int_{a}^{b} f(t) d t\right) \\
& \times\left(g(x)-\frac{(1 \pm \sqrt{1-\lambda})}{b-a} \int_{a}^{b} g(t) d t\right) d x .
\end{aligned}
$$

Secondly, substituting $m=k=1$ and $h_{1,1}(x)=h_{2,1}(x)=x$ in the inequality (11) yields

$$
\left(\mathbf{T}_{p+q-p q}\langle f, g\rangle\right)^{2} \leq \mathbf{T}_{p(2-p)}\langle f, f\rangle \mathbf{T}_{q(2-q)}\langle g, g\rangle \quad(p, q \in \mathbf{R})
$$

For $p=q=1$ the above inequality gives the same result as the pre-Grüss inequality (12) while for $p=q=0$ (or $p=q=2$ ) the Cauchy-Schwarz inequality is obtained. Also, for $p=q$ and $p(2-p)=w$, the Wagner inequality [7] is derived. An interesting case of the inequality (13) is when $\mathbf{T}_{p+q-p q}\langle f, g\rangle=\langle f, g\rangle$ (i.e., $1 / p+1 / q=1$ ), which reveals its importance in numerical integration formulas.

Moreover, since $p, q \in \mathbf{R}$, we can find the optimal parameters for the inequality (13). For this purpose, we consider two (positive) functions as follows:

$$
F_{1}(p)=\mathbf{T}_{q(2-q)}\langle g, g\rangle \mathbf{T}_{p(2-p)}\langle f, f\rangle-\left(\mathbf{T}_{p+q-p q}\langle f, g\rangle\right)^{2}
$$

and

$$
F_{2}(q)=\mathbf{T}_{p(2-p)}\langle f, f\rangle \mathbf{T}_{q(2-q)}\langle g, g\rangle-\left(\mathbf{T}_{p+q-p q}\langle f, g\rangle\right)^{2}
$$

The problem is now how to minimize $F_{1}(p)$ or $F_{2}(q)$. Since the final forms of both functions (14) and (15) are quadratic, i.e., as

$$
F_{1}(p)=A_{1}^{2}(q) p^{2}+B_{1}(q) p+C_{1}(q) \quad(q \text { constant })
$$


and

$$
F_{2}(q)=A_{2}^{2}(p) q^{2}+B_{2}(p) q+C_{2}(p) \quad(p \text { constant })
$$

to minimize, e.g., $F_{1}(p)$, after some computations, we finally get

$$
F_{1}^{\prime}(p)=0 \Rightarrow p=\frac{(b-a) \mathbf{T}_{q}\langle g, g\rangle \int_{a}^{b} f(t) d t+(q-1) \int_{a}^{b} f(t) g(t) d t \int_{a}^{b} g(t) d t}{(b-a) \mathbf{T}_{1}\langle g, g\rangle \int_{a}^{b} f(t) d t}
$$

where $q \in \mathbf{R}$. In particular, replacing $q=1$ in (16) gives the same as the pre-Grüss inequality.

Example 4 (A refinement of the Cauchy-Schwarz inequality via the generalized Wagner inequality) The following inequality for two sequences of real numbers $\left\{a_{i}\right\}_{i=1}^{n}$ and $\left\{b_{i}\right\}_{i=1}^{n}$ and a real parameter $\alpha \geq 0$ is known in the literature as the Wagner inequality [1, p.85]

$$
\left(\sum_{i=1}^{n} a_{i} b_{i}+\alpha \sum_{i=1}^{n} a_{i} \sum_{i=1}^{n} b_{i}\right)^{2} \leq\left(\sum_{i=1}^{n} a_{i}^{2}+\alpha\left(\sum_{i=1}^{n} a_{i}\right)^{2}\right)\left(\sum_{i=1}^{n} b_{i}^{2}+\alpha\left(\sum_{i=1}^{n} b_{i}\right)^{2}\right)
$$

This inequality is generalized in $[8, \mathrm{Re} .16]$ as follows:

$$
\begin{aligned}
& \left(\sum_{i=1}^{n} a_{i} b_{i}+\frac{p q-(p+q)}{n} \sum_{i=1}^{n} a_{i} \sum_{i=1}^{n} b_{i}\right)^{2} \\
& \quad \leq\left(\sum_{i=1}^{n} a_{i}^{2}+\frac{p(p-2)}{n}\left(\sum_{i=1}^{n} a_{i}\right)^{2}\right)\left(\sum_{i=1}^{n} b_{i}^{2}+\frac{q(q-2)}{n}\left(\sum_{i=1}^{n} b_{i}\right)^{2}\right) .
\end{aligned}
$$

Clearly, for $p=q \in\{\mathbf{R}-(0,2)\}$ and $p(p-2) / n=\alpha$ in (18), the inequality (17) is derived. Moreover, (18) is also a special case of the inequality (10).

Now, in order to obtain a refinement for the Cauchy-Schwarz inequality, we first assume in (18) that $p q=p+q$. This yields $q(q-2)=p(p-2) /(p-1)^{2}$, and therefore we wish to have

$$
\begin{aligned}
\left(\sum_{i=1}^{n} a_{i} b_{i}\right)^{2} & \leq\left(\sum_{i=1}^{n} a_{i}^{2}+\frac{p(p-2)}{n}\left(\sum_{i=1}^{n} a_{i}\right)^{2}\right)\left(\sum_{i=1}^{n} b_{i}^{2}-\frac{p(p-2)}{n(p-1)^{2}}\left(\sum_{i=1}^{n} b_{i}\right)^{2}\right) \\
& \leq \sum_{i=1}^{n} a_{i}^{2} \sum_{i=1}^{n} b_{i}^{2}
\end{aligned}
$$

which is equivalent to

$$
\begin{aligned}
& -\frac{p(p-2)}{n(p-1)^{2}} \sum_{i=1}^{n} a_{i}^{2}\left(\sum_{i=1}^{n} b_{i}\right)^{2}+\frac{p(p-2)}{n} \sum_{i=1}^{n} b_{i}^{2}\left(\sum_{i=1}^{n} a_{i}\right)^{2} \\
& -\frac{p^{2}(p-2)^{2}}{n^{2}(p-1)^{2}}\left(\sum_{i=1}^{n} a_{i}\right)^{2}\left(\sum_{i=1}^{n} b_{i}\right)^{2} \leq 0 .
\end{aligned}
$$


After some initial computations, the left-hand side of the above inequality is decomposable in terms of the variable $p$ in the form

$$
\lambda^{2} p(p-2)(p-1+\sqrt{S})(p-1-\sqrt{S}) \leq 0,
$$

in which

$$
\lambda^{2}=\frac{1}{n^{2}(p-1)^{2}}\left(\sum_{i=1}^{n} a_{i}\right)^{2}\left(n \sum_{i=1}^{n} b_{i}^{2}-\left(\sum_{i=1}^{n} b_{i}\right)^{2}\right) \geq 0,
$$

and

$$
S=\frac{\left(\sum_{i=1}^{n} b_{i}\right)^{2}\left(n \sum_{i=1}^{n} a_{i}^{2}-\left(\sum_{i=1}^{n} a_{i}\right)^{2}\right)}{\left(\sum_{i=1}^{n} a_{i}\right)^{2}\left(n \sum_{i=1}^{n} b_{i}^{2}-\left(\sum_{i=1}^{n} b_{i}\right)^{2}\right)} \geq 0 .
$$

Hence, by noting that $p \in\{\mathbf{R}-(0,2)\}$, then $p(p-2) \geq 0$ and the eligible region of the solution for the inequality (20) is $p \in\{\mathbf{R}-(0,2)\} \cap[1-\sqrt{S}, 1+\sqrt{S}]$. For instance, if

$$
\frac{\sum_{i=1}^{n} a_{i}^{2}}{\left(\sum_{i=1}^{n} a_{i}\right)^{2}} \leq \frac{\sum_{i=1}^{n} b_{i}^{2}}{\left(\sum_{i=1}^{n} b_{i}\right)^{2}}
$$

in (19) then it is directly concluded that $S \geq 1$ in (21) and conversely. Therefore, the solution of (20) would be either $p \in[2,1+\sqrt{S}]$ or $p \in[1-\sqrt{S}, 0]$. This means that the refinement (19) is valid for any $p \in[2,1+\sqrt{S}]$ or $p \in[1-\sqrt{S}, 0]$ provided that the condition (22) holds.

Similarly, the latter result holds for the continuous case and we have

Corollary 5 Iff, $g \in C([a, b], \mathbf{R})$, then the following refinement for the Cauchy-Bunyakovsky inequality holds:

$$
\begin{aligned}
\left(\int_{a}^{b} f(x) g(x) d x\right)^{2} \leq & \left(\int_{a}^{b} f^{2}(x) d x+\frac{p(p-2)}{b-a}\left(\int_{a}^{b} f(x) d x\right)^{2}\right) \\
& \times\left(\int_{a}^{b} g^{2}(x) d x-\frac{p(p-2)}{(b-a)(p-1)^{2}}\left(\int_{a}^{b} g(x) d x\right)^{2}\right) \\
\leq & \int_{a}^{b} f^{2}(x) d x \int_{a}^{b} g^{2}(x) d x
\end{aligned}
$$

provided that $p \in\{\mathbf{R}-(0,2)\} \cap\left[1-\sqrt{S^{*}}, 1+\sqrt{S^{*}}\right]$ where

$$
S^{*}=\frac{\left(\int_{a}^{b} g(x) d x\right)^{2}\left((b-a) \int_{a}^{b} f^{2}(x) d x-\left(\int_{a}^{b} f(x) d x\right)^{2}\right)}{\left(\int_{a}^{b} f(x) d x\right)^{2}\left((b-a) \int_{a}^{b} g^{2}(x) d x-\left(\int_{a}^{b} g(x) d x\right)^{2}\right)} \geq 0 .
$$




\section{Author details}

${ }^{1}$ Department of Mathematics, K.N. Toosi University of Technology, P.O. Box 16315-1618, Tehran, Iran. ${ }^{2}$ Department of Mathematics, King Abdulaziz University, P.O. Box 80203, Jeddah, 21589, Saudi Arabia.

\section{Acknowledgements}

The work of the first author is supported by the grant from 'Iran National Science Foundation' No. 91002576. The second author gratefully acknowledges the support from the Deanship of Scientific Research (DSR) at King Abdulaziz University during this research.

Received: 17 July 2012 Accepted: 1 October 2012 Published: 17 October 2012

\section{References}

1. Mitrinovic, DS, Pecaric, JE, Fink, AM: Classical and New Inequalities in Analysis. Kluwer Academic, Dordrecht (1993)

2. Callebaut, DK: Generalization of the Cauchy-Schwarz inequality. J. Math. Anal. Appl. 12, 491-494 (1965)

3. Dragomir, SS: A survey on Cauchy-Bunyakovsky-Schwarz type discrete inequalities. J. Inequal. Pure Appl. Math. 4(3), Article ID 63 (2003)

4. Dragomir, SS, Cho, YJ, Pearce, CEM, Kim, SS: Cauchy-Schwarz functionals. Bull. Aust. Math. Soc. 62, $479-491$ (2000)

5. Dragomir, SS, Cho, YJ, Kim, SS: Some new results related to Bessel and Grüss inequalities in 2-inner product spaces and applications. Bull. Korean Math. Soc. 42, 591-608 (2005)

6. Masjed-Jamei, M, Dragomir, SS, Srivastava, HM: Some generalizations of the Cauchy-Schwarz and the Cauchy-Bunyakovsky inequalities involving four free parameters and their applications. Math. Comput. Model. 49 1960-1968 (2009)

7. Wagner, SS: Not. Am. Math. Soc. 12, 220 (1965)

8. Masjed-Jamei, M: A functional generalization of the Cauchy-Schwarz inequality and some subclasses. Appl. Math. Lett. 22, 1335-1339 (2009)

9. Masjed-Jamei, M, Qi, F, Srivastava, HM: Generalizations of some classical inequalities via a special functional property. Integral Transforms Spec. Funct. 21, 327-336 (2010)

10. Chebyshev, PL: Sur les expressions approximative des integrals par les auters prises entre les mêmes limites. Proc. Math. Soc. Charkov 2, 93-98 (1882)

11. Grüss, G: Uber das Maximum des absoluten Betrages von $\frac{1}{b-a} \int_{a}^{b} f(x) g(x) d x-\frac{1}{(b-a)^{2}} \int_{a}^{b} f(x) d x \int_{a}^{b} g(x) d x$. Math. Z. 39 215-226 (1935)

\section{Submit your manuscript to a SpringerOpen ${ }^{\ominus}$ journal and benefit from:}

- Convenient online submission

- Rigorous peer review

- Immediate publication on acceptance

- Open access: articles freely available online

- High visibility within the field

- Retaining the copyright to your article 